VOL. 12 (1975), 49-72.

\title{
A notion of limit for enriched categories
}

\section{Francis Borceux and G.M. Kelly}

For a $V$-category $B$, where $V$ is a symmetric monoidal closed category, various limit-like notions have been recognized: ordinary limits (in the underlying category $B_{0}$ ) preserved by

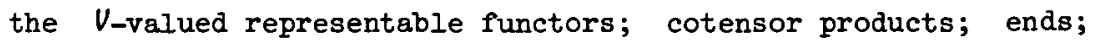
pointwise Kan extensions. It has further been recognized that, to be called complete, $B$ should admit all of these; for which it suffices to demand the first two. Hitherto, however, there has been no single limit-notion of which all these are special cases, and particular instances of which may exist even when $B$ is not complete or even cotensored. In consequence it has not been possible even to state, say, the representability criterion for a $V$-functor $T: B \rightarrow V$, or even to define, say, pointwise Kan extensions into $B$, except for cotensored $B$. (It is somewhat as if, for ordinary categories, we had the notions of product and equalizer, but lacked that of general limit, and could not discuss pullbacks in the absence of products.) In this paper we provide such a general limit-notion for $V$-categories.

\section{Introduction}

$V$ is a symetric monoidal closed category with tensor product $\theta$, identity object $I$, and internal-hom $[$,$] ; recall that [X, Y]$ is also the $V$-valued-hom $V(X, Y)$ for the $V$-category $V$. We write $B_{0}$ for the ordinary category underlying the V-category $B$; in particular,

Received 25 September 1974. 
the ordinary category underlying $V$ is $V_{0}$. For the basic facts about $V$-categories we refer to Eilenberg and Kelly [4], Kelly [5], Day and Kelly [2], and Dubuc [3].

All our classes of objects (for a category or for a V-category) and our classes of morphisms (for an ordinary category) are sets; small sets are those in some universe chosen once for all; Set is the category of small sets; an ordinary category is locally small if its hom-sets are small, that is, if it is a Set-category; a V-category is small if its set of objects is small; an ordinary category is small if it is a small Set-category, that is, if its set of morphisms is small.

It has been recognized [2] that, for instance, the product of objects $B$ and $C$ in a $V$-category $B$ should be an object $B \times C$, with projections in $B_{0}$ to $B$ and to $C$, such that for each $A$ the projections define an isomorphism

$$
B(A, B \times C) \cong B(A, B) \times B(A, C)
$$

in $v_{0}$, the right side being the ordinary product in $V_{0}$. Applying the underlying-set functor $V_{0}(I,-)$ from $V_{0}$ to (possibly large) sets, we deduce that the projections also define an isomorphism

$$
\mathrm{B}_{\mathrm{O}}(A, B \times C) \cong \mathrm{B}_{0}(A, B) \times \mathrm{B}_{0}(A, C)
$$

of sets, so that $B \times C$ is a fortiori the ordinary product of $B$ and $C$ in $B_{0}$. In general, however, (1.2) is far from implying (1.1); for instance, when $V$ is graded abelian groups, $B_{0}(A, B)$ contains only an infinitesimal part of the information in $B(A, B)$. We call $B \times C$ satisfying (1.1) the product in $B$; if it merely satisfies (1.2) it is what is classically called the product in $B_{0}$. The latter is, of itself, clearly not the proper object of interest for $V_{\text {-categories. Another way }}$ of saying that $B \times C$ is the product in $B$ is to say that it is the product in $B_{0}$ and that it is preserved by the $V$-valued representables $B(A,-): B_{0} \cdot V_{0}$ for all $A$. Similar remarks apply to the limit in $B$ and the limit in $B_{0}$ of any ordinary functor $Q: K \rightarrow B_{0}$. (Some authors speak of strong and weak limits, or of V-limits and limits.) 
The existence in $B$ of such limits for all small $K$ and $a l l Q$ is not, however, enough to allow us to prove the $V$-analogues of those results that, in the case of ordinary categories, would follow from completeness of $B$. We need also the notion [5] of the cotensor product $[X, B] \in B$ of $X \in V$ with $B \in B$, namely the object giving a $V$-representation

$$
B(A,[X, B]) \cong[X, B(A, B)] \text {. }
$$

When $V=$ Set this is just the product of $X$ copies of $B$, or the $X$-th power of $B$, and falls under the ordinary notion of limit. For a general $V$, however, it is an independent limit-like notion. We call $B$ cotensored if $[X, B]$ exists for all $X$ and $B ; \quad V$ itself is always cotensored, $[X, B]$ then being the internal-hom.

$B$ is called complete in [2] if it is cotensored and admits all small limits; various results are established in [2], [3], and [1] for such complete $B$. Other limit-like notions of great utility are also introduced: ends in [2], pointwise Kan extensions in [2] and [3]. The former are shown to exist (for a small domain) if $B$ is cotensored and admits certain limits; the latter are not even defined (except for ordinary categories - see Mac Lane [6], p. 239) unless B is cotensored ([3], p. 54).

For ordinary categories all limits are, in the complete case, combinations of products and equalizers; but in the incomplete case a particular limit may exist although products do not. For V-categories, replacing "product" by "cotensor product" and "equalizer" by "limit in $B$ ", the analogue of the first clause is true but that of the second is meaningless - for there is no general limit-notion containing cotensor product and limit in $B$ as special cases. Our purpose is to provide such a notion.

\section{Outline}

Ordinary limits involve a functor $G: A \rightarrow B$ and a cone over it; a cone involves the idea of a constant functor $A \rightarrow I \rightarrow B$ where $I$ is the unit category; and the last is lacking for $V$-categories. We still have the unit $V_{\text {-category }} I$ with one object 0 and with $I(0,0)=I$, and $V$-functors $I \rightarrow B$ still correspond to objects of $B$; but there is no canonical $V$-functor $A+I$. The limit-notion we propose for a 
$V$-category $B$ involves a V-functor $G: A \rightarrow B$ and, in place of a constant functor, an arbitrary $V$-functor $F: A \rightarrow V$.

To these we assign as our "limit" (when it exists) an object $\{F, G\}$ of $B$. If $B$ admits the cotensor products $[E A, G A]$ for each $A \in A$, it will be the object

$$
\{F, G\}=\int_{A}[F A, G A],
$$

existing whenever the integral $(=$ end) on the right exists. So the name we give it is mean cotensor product of $F$ and $G$. To define it when the indicated cotensor products do not exist, observe that applying $B(B,-)$ to (2.I) gives

$$
B(B,\{F, G\})=\int_{A}[F A, B(B, G A)] ;
$$

here the cotensor products on the right certainly exist, being merely internal-homs in $V$. If now the integral on the right exists for each $B$, it is a $V$-functor of $B$; if moreover this $V$-functor admits a $V$-representation as in $(2.2)$, we call the representing object $\{F, G\}$; which is then said to exist.

All the other limit-notions are now special cases. Taking $A=I$ and identifying for example $G: I \rightarrow B$ with an object $G$ of $B$, we see that $\{F, G\}$ becomes the ordinary cotensor product $[F, G]$. Taking $A$ to be the free $V$-category on the ordinary category $K$ (which exists if $K$ is locally small and $V_{0}$ admits small copowers), and taking $G: A \rightarrow B$ to be the $V$-functor induced by the ordinary functor $Q: K \rightarrow B_{0}$, we do now have a canonical $V$-functor * : $A \rightarrow V$ (induced by the functor $K+V_{0}$ constant at $I)$, and $\{*, G\}$ is precisely the limit of $Q$ in $B$. For $A=D^{\mathrm{OP}} \otimes D$ and $F=$ hom $_{D}: D^{\mathrm{OP}} \otimes D \rightarrow V$, it turns out that $\left\{\mathrm{Hom}_{D}, G\right\}$ is precisely the end $\int_{D} G(D, D)$. Finally, if we have a V-functor $K: A \rightarrow C$ and set $F=C(C, K-)$, we recover the pointwise Kan extension of $G$ along $K$ in the form

$$
\left(\operatorname{Ran}_{K} G\right) C=\{C(C, K-), G\}
$$


The dual notion involves $V$-functors $H: A^{\circ p} \rightarrow V$ and $G: A \rightarrow B$, and is the mean tensor product $H \star G \in B$; defined by

$$
H * G=\left\{H, G^{\mathrm{op}}\right\}
$$

where $G^{\text {OP }}: A^{O P} \rightarrow B^{O P}$, and given by

$$
\bar{H} * G=\int^{A} H A \otimes G A
$$

when the tensor products in the integrand exist.

The above approach uses the (very special) end on the right of (2.2) to define $\{F, G\}$; since ends are a special case of $\{F, G\}$ this offends against economy or aesthetics; whence we give an account requiring no prior knowledge of ends. To do so we have only to recognize that we get something equivalent to $(2.2)$ if we apply $v_{0}(X,-)$ to both sides, for an arbitrary $X \in V$, and then re-write the right side in terms of a set of $v$-natural transformations.

\section{The main definition}

The mean cotensor product $\{F, G\}$ of the $V$-functors $F: A \rightarrow V$ and $G: A \rightarrow B$ is said to exist if there are an object $\{F, G\}$ of $B$ and $a$ $V$-natural transformation

$$
\lambda: F+B(\{F, G\}, G-)
$$

such that, for each $B \in B$ and each $X \in V$, the function

$$
\pi: V_{0}(X, B(B,\{F, G\})\} \rightarrow V_{-\operatorname{nat}}(F,[X, B(B, G-)])
$$

is bijective; here the codomain of (3.2) is the set of $V$-natural transformations $F \rightarrow\left[X, B\left(B, G_{-}\right)\right]$and $\pi$ sends $f$ to the composite (3.3) $F \underset{\lambda}{\longrightarrow} B(\{F, G\}, G) \underset{B(B,-)}{\longrightarrow}[B(B,\{F, G\}), B(B, G-)]$

$$
\overrightarrow{[f, 1]}[X, B(B, G)] \text {. }
$$

Taking $X=I$ in (3.2) and simplifying, we get a bijection

$$
\pi^{\prime}: B_{0}(B,\{F, G\})+V-\operatorname{nat}(F, B(B, G)) \text {, }
$$

sending $g$ to the composite 


$$
F \underset{\lambda}{\longrightarrow} B(\{F, G\}, G-) \underset{B(g, I)}{\longrightarrow} B(B, G-) .
$$

This exhibits $\{F, G\}$ and $\lambda$ as the representing object and the characteristic morphism for the functor given by the codomain of (3.4), and shows their uniqueness, when they exist, to within a unique isomorphism.

It is clear that $V$-natural transformations $\alpha: F^{\prime} \rightarrow F$ and $B: G \rightarrow G^{\prime}$ induce, when the domain and codomain exist, a unique

$$
\{\alpha, \beta\}:\{F, G\} \rightarrow\left\{F^{\prime}, G^{\prime}\right\}
$$

with the appropriate relation to (3.2).

The following Yoneda-type result generalizes [2], 3.5, reducing to it when $B$ is cotensored.

THEOREM 3.1. Let $G: A \rightarrow B$ be a V-finctor and let $A \in A$. We have

$$
\{A(A,-), G\}=G A,
$$

the corresponding $\lambda$ being $G: A(A,-) \rightarrow B(G A, G-)$.

Proof. Use the lower-level V-Yoneda-Lemma of [4], Chapter I, Theorem 8.6 to replace the codomain of (3.2) by $v_{0}(I,[X, B(B, G A)])$, isomorphic to $v_{0}(X, B(B, G A))$.

\section{Cotensor products}

As we said in $\$ 2$, when $A$ is the unit $V$-category $I$ we identify $F$ and $G$ with objects of $V$ and of $B$ respectively, and call $\{F, G\}$ the cotensor product $[F, G]$ of these objects. In this case the codomain of (3.2) is just $V_{0}(F,[X, B(B, G)])$; replacing this by its isomorph $V_{0}(X,[F, B(B, G)])$ replaces $\pi$ in (3.2) by $V_{0}(1,8)$, where

$$
\boldsymbol{8}: B(B,[F, G]) \rightarrow[F, B(B, G)]
$$

is the composite

(4.2) $B(B,[F, G]) \underset{B(-, G)}{\vec{B}}[B([F, G], G), B(B, G)] \underset{[\lambda, 1]}{\longrightarrow}[F, B(B, G)]$.

Since (3.2) is an isomorphism for all $X$ if and only if (4.1) is an isomorphism, we conclude that the cotensor product $[F, G]$ exists precisely where $[F, G]$ and $\lambda: F \rightarrow B([F, G], G)$ provide a 
V-representation of the V-functor $B \mapsto[F, B(B, G)]$. This is the definition given in [5].

When $B$ is cotensored, that is when $[F, G]$ exists for all $F$ and $G,(4.1)$ exhibits $[-, G]$ as the $V$-left-adjoint of $B(-, G): B^{\text {op }} \rightarrow V$. In this case, by the results of [5], $F, G \mapsto[F, G]$ is a $V$-functor $V^{O P} \otimes B \rightarrow B$ in such a way that $(4.1)$ is $V$-natural in all variables.

Similarly for the dual notion of tensor product $H \otimes G \in B$ of $H \in V$ and $G \in B$, for which $(4.1)$ becomes

$$
B(H \otimes G, B) \cong[H, B(G, B)] \text {. }
$$

of course $V$ itself is tensored and cotensored, $H \otimes G$ and $[F, G]$ being the usual tensor product and internal-hom.

\section{The weaker definition}

Returning to a general $A$, we call an object $\{F, G\}$ of $B$ along with the $V$-natural $\lambda$ of (3.1) the weak mean cotensor product of $F$ and $G$ if the $\pi^{\prime}$ of (3.4) is a bijection; we then say that $\{F, G\}$ exists weakly. Thus by $\$ 3$ existence implies weak existence; the converse is false in general. The distinction is analogous to that of $\$ 1$ between limits in $B$ and limits in $B_{0}$; and, as there, weak existence is not in itself of interest in the context of $V$-categories. We introduce it only because in certain cases it implies existence; and it is easier to verify.

PROPOSITION 5.1. Weak existence implies existence if $B$ is tensored, and thus in particular if $B=V$.

Proof. Replacing $B$ by $X \otimes B$ in (3.4) gives (3.2).

PROPOSITION 5.2. Weak existence implies existence, for any $B$, if $V$ is the category of algebras for a monoidal monad on Set; for excomple, if $V=$ Set, or $\mathrm{Ab}$, or R-Mod for a commutative ring $R$.

Proof. In these cases the underlying-set functor $v_{0}(I,-)$ is faithful and reflects isomorphisms; and $[X, Y]$ is made from $V_{0}(X, Y)$ by defining the operations element-wise.

Moreover $V_{0}$ here is locally small, whence $B_{0}$ too is so. Since 
(3.4) is a bijection by hypothesis, its codomain is a small set. Elementwise operations on $V$-natural transformations (which, since the underlyingset functor is faithful, are just the natural ones) turn this latter set into an object $Y$ of $V$, and $\pi^{\prime}$ is easily seen to be a $V_{0}$-morphism $B(B,\{F, G\}) \rightarrow Y$. It is an isomorphism because $V_{0}(I,-)$ reflects isomorphisms.

Finally the codomain of $(3.2)$ is easily identified with $v_{0}(X, Y)$, whereupon $\pi$ becomes $V_{0}\left(1, \pi^{\prime}\right)$, an isomorphism because $\pi^{\prime}$ is.

In the case $V=$ Set we have introduced nothing transcending the ordinary notion of limit; for we have:

THEOREM 5.3. In the case $V=$ Set write $I$ not only for the onepoint set but also for the functor $I \rightarrow$ Set sending the unique object 0 of $I$ to $I$. Let $I / F$ denote the comma category of $I: I \rightarrow$ Set and $F: A \rightarrow$ Set, commonly called the category of elements of $F$; and let $d: I / F \rightarrow A$ be the projection. Then for $G: A \rightarrow B$ we have

$$
\{F, G\}=\lim G d \text {, }
$$

either side existing if the other does.

Proof. By Proposition 5.2 weak existence of $\{F, G\}$ coincides with existence. The codomain of (3.4) is easily identified with the set of conves over $G d$ with vertex $B$.

In analogy with the case of limits in $B$ discussed in $\$ 1$, existence reduces via the representables to weak existence in $V$ :

PROPOSITION 5.4. $\{F, G\}$ and the $\lambda$ of (3.1) constitute the mean cotensor product of $F: A \rightarrow V$ and $G: B \rightarrow V$ if and only if, for each $B \in B$, the object $B(B,\{F, G\})$ and the composite V-natural transformation

$$
F \vec{\lambda} B(\{F, G\}, G-) \underset{B(B,-)}{\longrightarrow}[B(B,\{F, G\}), B(B, G-)]
$$

constitute weakly the mean cotensor product $\{F, B(B, G-)\}$ of $F: A \rightarrow V$ and $B(B, G-): A \rightarrow V$.

Proof. Compare (3.5) with (3.3). 


\section{Preservation, representability, adjoints}

Let $\{F, G\}$ along with the $\lambda$ of (3.1) be the mean cotensor product of $F: A \rightarrow V$ and $G: A \rightarrow B$. A $V$-functor $T: B \rightarrow C$ is said to preserve $\{F, G\}$ if $T\{F, G\}$, along with the $U$-natural transformation

$$
F \underset{\lambda}{\longrightarrow} B(\{F, G\}, G-) \underset{T}{\longrightarrow} C(T\{F, G\}, T G-)
$$

(which we write as $T \cdot \lambda$ ), is the mean cotensor product $\{F, T G\}$ of $F$ with $T G: A+C$.

When $A=I$ this agrees with the definition of "preserving cotensor products" given in [5].

If $\{F, G\}$ exists weakly and if $T\{F, G\}$ and $T \cdot \lambda$ constitute $\{F, T G\}$ weakly, we say that $T$ weakly preserves $\{F, G\}$.

THEOREM 6.1. The representables $B(B,-): B \rightarrow V$ preserve any $\{F, G\}$ that exists.

Proof. Proposition 5.4 and Proposition 5.1.

By a left adjoint for a $V$-functor we mean of course a $V$-leftadjoint. Recall from [5] that the $U$-functor $T: B \rightarrow C$ has a left adjoint if and only if $C\left(C, T_{-}\right)$is representable for each $C \in C$.

THEOREM 6.2. If $T: B \rightarrow C$ has a left adjoint it preserves any $\{F, G\}$ that exists.

Proof. $\{F, G\}$ is, by Theorem 6.1, preserved by $C(C, T-)$; the result follows on applying Proposition 5.4 to $T\{F, G\}$ and $T \cdot \lambda$.

The notion of mean cotensor product enables us to give for the first time a necessary and sufficient condition for representability of a $V$-functor, without extraneous conditions. (The necessary and sufficient condition for the existence of a left adjoint, given in [3], Theorem I.4.1, does not in general give a criterion for representability.) To cover representability and adjoints in one theorem, it is convenient to prove the following:

THEOREM 6.3. Let $G: A \rightarrow B$ be a V-finctor, let $B \in B$, and denote by 1 the identity $1: A \rightarrow A$. Then the following are equivalent:

(i) $B(B, G): A \rightarrow V$ is representable;

(ii) $\{B(B, G-), 1\}$ exists and is preserved by $G$; 
(iii) $\{B(B, G-), 1\}$ exists weakly and is weakly preserved by G.

Then the representing object is $\{B(B, G), I\}$ and the corresponding $\lambda: B\left(B, G_{-}\right) \rightarrow A\left(\left\{B\left(B, G_{-}\right), 2\right\},-\right)$ provides the representation, being in fact an isomorphism.

Proof. (i) implies (ii) by Theorems 3.1 and 6.1 , and (ii) implies (iii) trivially. To prove that (iii) implies (i) we construct, under the hypotheses of (iii), an inverse of $\lambda$.

Since $G\{B(B, G-), I\}$ and $G \cdot \lambda$ weakly constitute $\{B(B, G-), G\}$, there is a unique $g: B \rightarrow G\{B(B, G), 1\}$ such that the $V$-natural transformation

(6.2) $B\left(B, G_{-}\right) \underset{\lambda}{\longrightarrow} A\left(\left\{B\left(B, G_{-}\right), 1\right\},-\right) \underset{G}{\rightarrow} B\left(G\left\{B\left(B, G_{-}\right), 1\right\}, G_{-}\right)$

$$
\overrightarrow{B(g, 1)} B(B, G-)
$$

is the identity. Writing $\mu$ for the composite of the last two factors in (6.2) we have $\mu \lambda=1$. The other composite

$$
\lambda \mu: A\left(\left\{B\left(B, G_{-}\right), 1\right\},-\right) \rightarrow A(\{B(B, G-), 1\},-)
$$

is, by the ordinary $V$-Yoneda-Lemma, of the form $A(f, 1)$ for some endomorphism $f$ of $\{B(B, G-), 1\}$. Since $\mu \lambda=1$ we have $\lambda \mu \lambda=\lambda$ or $A(f, 1) \lambda=A(1,1) \lambda$. Using the definition of weak existence of $\left\{B\left(B, G_{-}\right), 1\right\}$ we conclude from this that $f=1$ and hence that $\lambda \mu=1.0$

Taking $B=V$ and $B=I$ in Theorem 6.3, we get:

THEOREM 6.4. For a $V$-functor $G: A \rightarrow V$ the following are equivalént:

(i) $G$ is representable;

(ii) $\{G, 1\}$ exists and is preserved by $G$;

(iii) $\{G, 1\}$ exists weakly and is weakly preserved by $G$.

Then $\lambda$ is an isomorphism and the representation is $\lambda: G \rightarrow A(\{G, 1\},-)$.

Quantifying over $B$ in Theorem 6.3 gives:

THEOREM 6.5. For $a$ V-finctor $G: A+B$ the following are equivalent: 
(i) $G$ has a left adjoint;

(ii) for each $B,\{B(B, G-), 1\}$ exists and is preserved by $G$;

(iii) for each $B,\{B(B, G), 1\}$ exists weakly and is weakly preserved by $G$.

Then the left adjoint $S$ of $G$ is given on objects by $S B=\{B(B, G-), 1\}$.

\section{Ends}

Given a $V$-functor $K: D^{\circ \mathrm{P}} \otimes D \rightarrow B$ we write $\mathrm{Hom}_{D}: D^{\circ \mathrm{p}} \otimes D \rightarrow V$ for the $U$-valued Hom-functor and define the end of $K$, written $\int_{D} K(D, D)$, by

$$
\int_{D} K(D, D)=\left\{\mathrm{Hom}_{D}, K\right\}
$$

whenever the right side exists: in which case we say that the end (also called the integral) exists. The dual notion is the co-end or co-integral

$$
\int^{D} K(D, D)=\text { Hom }_{D} * K
$$

$$
\text { The corresponding } \lambda: \operatorname{Hom}_{D}\left(D^{\prime}, D^{\prime \prime}\right)+B\left(\int_{D} K(D, D), K\left(D^{\prime}, D^{\prime \prime}\right)\right)
$$

corresponds by the V-Yoneda-Lemma to a V-natural $\mu: \int_{D} K(D, D) \rightarrow K\left(D^{\prime}, D^{\prime}\right)$. (This is of course the "extraordinary"

$V$-naturality of [4], Chapter III, \$5.) Weak existence of (7.1) merely asks that $\mu$ be terminal among $V$-natural $v: C \rightarrow K\left(D^{\prime}, D^{\prime}\right)$; true existence demands a similar terminal property of $B(B, \mu)$ among the $B(B, \nu)$ for each $B \in B$. Our definition of end, therefore, agrees precisely with the original definition in [2], including the two levels at which it may exist. Once again, we have no serious interest in weak existence except insofar as it implies existence. Further, preservation in our sense of $\left\{\mathrm{Hom}_{D}, K\right\}$ by $T: B \rightarrow C$ coincides with preservation of the end $\int_{D} K(D, D)$ as defined 
in [2]. We leave the reader to check the details.

Conversely, we get the expression (2.1) of our Outline above, for $\{F, G\}$ as an end, when the necessary cotensor products exist:

PROPOSITION 7.1. Let $F: A \rightarrow V$ and $G: A \rightarrow B$, and let the cotensor product $[F A, G A]$ exist for each $A \in A$; which it certainly does if $B$ is cotensored and in particular if $B=V$. Then we have

$$
\{F, G\}=\int_{A}[F A, G A],
$$

either side existing if the other does.

Proof. Writing in for clarity the variable $A \in A$ in which there is to be $V$-naturality, and admitting extraordinary $V$-naturality into our formulas, we transform as follows the codomain of (3.2) using the cotensor products:

$$
\begin{aligned}
v-\operatorname{nat}(F A,[X, B(B, G A)]) & \cong v-\operatorname{nat}(X,[F A, B(B, G A)]) \\
& \cong v-\operatorname{nat}(X, B(B,[F A, G A])) .
\end{aligned}
$$

After similar transformations of the $\lambda$ of (3.1) and the $\pi$ of (3.2), the result is immediate.

For ends it is particularly easy to establish the $V$-functoriality of $\int_{D} K(D, D, E)$ in the extra variable $E$, where here $K: D^{\circ \mathrm{p}} \otimes D \otimes E \rightarrow B$; related results about the $V$-naturality of induced morphisms; and the Fubini Theorem. These things are treated in [2], \$3, and in more detail in [1], \$2; the proofs can hardly be improved and we just take these results over.

In particular, for $F: A \rightarrow V$ and $G: A+B$,

$$
\left\{F, B\left(B, G_{-}\right)\right\}=\int_{A}[F A, B(B, G A)]
$$

is a V-functor in $B$ if it exists for all $B$. We can now get the definition of $\{F, G\}$ given in (2.2) of our Outline above.

THEOREM 7.2. $\{F, G\}$ exists if and only if (7.4) exists for each $B \in B$ and is representabie as a $V$-functor $B^{\circ P} \rightarrow V$; the representing object is $\{F, G\}$, so that we have 


$$
p: B(B,\{F, G\}) \cong \int_{A}[F A, B(B, G A)]
$$

and the characteristic map $I \rightarrow \int_{A}[F A, B(\{F, G\}, G A)]$ is just the transform of $\lambda$.

Proof. If $\{F, G\}$ exists then, by Theorem 6.1, $\{F, B(B, G)\}$ exists and is given by $B(B,\{F, G\})$ along with $B(B,-) \cdot \lambda$. It is easily checked that the isomorphism (7.5) is given in terms of $\lambda$ as stated in the theorem, and is therefore $V$-natural in $B$, as desired.

For the converse we have only to apply $v_{0}(X,-)$ to $(7.5)$ and transform the right side into the codomain of (3.2), at the same time checking that the isomorphism becomes the $\pi$ given by (3.3).

The following lemma is "classical" and has been used in many special cases, for instance in [5], 3.3 and 3.5; but there does not seem to be an explicit reference in the literature:

LEMMA 7.3. If the $V$-finctor $T: A^{\mathrm{OP}} \otimes B \rightarrow V$ is such that we have for each fixed $B$ a representation $p: A(A, S B) \cong T(A, B)$ of $T(-, B)$, then there is a unique way of making $S$ into a V-finctor $S: B \rightarrow A$ rendering $p$ V-natural in $B$.

Proof. By [4], Chapter III, Proposition 7.9, $p$ is V-natural in $B$ if and only if the characteristic morphism $\alpha: I \rightarrow T(S B, B)$ is so. The latter says that $S_{B B^{\prime}}: B\left(B, B^{\prime}\right) \rightarrow A\left(S B, S B^{\prime}\right)$ followed by $[\alpha, 1] T\left(-, B^{\prime}\right)$ should equal $[\alpha, 1] T(S B,-)$. But $[\alpha, 1] T\left(-, B^{\prime}\right)$ equals the isomorphism ip $: A\left(S B, S B^{\prime}\right) \rightarrow T\left(S B, B^{\prime}\right) \rightarrow\left[I, T\left(S B, B^{\prime}\right)\right]$. Thus we are forced to define $S_{B B^{\prime}}$ as $p^{-1} i^{-1}[\alpha, 1] T(S B,-)$. We then easily verify the axioms for a V-functor.

The lemma, with Theorem 7.2 , at once allows us to transfer the "dependence on extra variables" results from ends to arbitrary $\{F, G\}$ :

PROPOSITION 7.4. If $F: A \otimes C \rightarrow V$ and $G: A \otimes D \rightarrow B$, and if $\{F(-, C), G(-, D)\}$ exists for each $C$ and $D$, there is a vonique way of making this into a $V$-functor $C^{O p} \otimes D \rightarrow B$ that renders the $p$ of $(7.5)$ $V$-natural in $C$ and $D$. 
We leave to the reader the easy verifications in:

PROPOSITION 7.5. The $\{\alpha, \beta\}$ of (3.6) is $V$-natural in any extra variables in $F$ and $G$. The isomorphism (3.7) is V-natural in $A$ and in any extra variables in $G$.

\section{Kan extensions}

The classical notion of Kan extension (as distinct from that of pointwise Kan extension) can be defined in any 2-category. In our 2-category of $V$-categories, $V$-functors, and $V$-natural transformations, it is as follows.

Given $V$-functors $K: A \rightarrow D$ and $G: A \rightarrow B$, we understand by the right Kan extension of $G$ along $K$ a $V$-functor $\operatorname{ran}_{K} G: D \rightarrow B$ together with a $V$-natural transformation

$$
\varepsilon:\left(\operatorname{ran}_{K} G\right) K \rightarrow G
$$

such that, for each $V$-functor $P: D \rightarrow B$, the function

$$
\theta: V_{-\operatorname{nat}}\left(P, \operatorname{ran}_{K} G\right) \rightarrow V_{-\operatorname{nat}}(P K, G)
$$

is a bijection; where $\theta$ sends $\alpha$ to the composite

$$
P K \underset{\alpha K}{\longrightarrow}\left(\operatorname{ran}_{K} G\right) K \cdot \vec{\varepsilon} G \text {. }
$$

Clearly $\operatorname{ran}_{K} G$ and $\varepsilon$, if they exist, are unique to within a unique isomorphism.

We say that the right Kun extension above is respected by a V-functor $T: B \rightarrow C$ if $T\left(\operatorname{ran}_{K} G\right)$ and $T \varepsilon$ constitute $\operatorname{ran}_{K} T G$. It is classical ([3], Proposition 1.4.2) that right Kan extensions are respected by any $T$ that has a left adjoint, but need not be respected by the representables $B(B,-): B \rightarrow V$, even in the case $V=$ Set .

Where we say that $T$ respects a Kan extension, most authors say that $T$ preserves it. The latter term is unfortunate and leads to confusion; in the case $v=$ Set, any limit notion should be preserved by representables, and yet the usual terminology suggests otherwise for Kan extensions. The truth of the matter is that the Kan extension lives not in $B$ but in the functor-category $[D, B]$; it is a limit indeed, but in 
[D,B] not in $B$; to say that it is reopected by $T$ is to say that it is preserved by $[1, T]:[D, B]+[D, C]$; if $T$ has a left adjoint so does $[1, T]$, so that $[1, T]$ preserves and $T$ respects; but if $T$ is the representable $B(B,-)$ it is not in general the case that $[1, T]$ has a left adjoint.

The point is sufficiently illustrated by considering, again in the case $V=$ Set, an ordinary limit in a functor category $[D, B]$. It is said to exist pointwise if for each $D \in D$ the corresponding limit exists in $B$; the totality of these then automatically constitutes the limit in $[D, B]$. Yet a fortuitous relation between $D$ and $B$ may allow a limit to exist without existing pointwise. Mere existence requires a certain completeness of $[D, B]$; pointwise existence requires a certain completeness of $B$. The limit exists pointwise if it exists and is preserved by the evaluations $E_{D}:[D, B] \rightarrow B$; this is the same as asking it to be preserved by $[1, B(B,-)]$ for each $B \in B$, or to be respected by the representables $B(B,-)$, as an easy calculation shows.

This suggests our definition below of pointwise Kan extension; in the case $V=$ Set it reduces to that of MaC Lane ([6], p. 240); for a general $V$ it has been defined by Dubuc ( $[3]$, p. 54) only for cotensored $B$, where there is an integral formula for it; our notion of mean cotensor product extends this formula to the case of any $B$ whatsoever. Results similar to those of this section have been given by Zandarin-Vandenbeyvanghe [7].

We remark finally that we know of no important result involving Kan extensions where these are not pointwise; pointwiseness, if not explicit in the hypotheses, is at least a consequence of them. It is convenient to indicate notationally that a right Kan extension exists pointwise by using a capital $R$ and writing $\operatorname{Ran}_{K} G$ in place of $\operatorname{ran}_{K} G$.

We say, then, that $\operatorname{Ran}_{K} G: D \rightarrow B$ together with $\varepsilon:\left(\operatorname{Ran}_{K} G\right) K \rightarrow G$ is the pointwise might Kon extension of $G$ along $K$ if we have (i) and (ii):

(i) $\operatorname{Ran}_{K} G$ with $\varepsilon$ is the right Kan extension of $G$ along $K$;

(ii) this right Kan extension is respected by the representable $B(B,-): B \rightarrow V$ for each $B \in B$.

We express the existence of this pointwise Kan extension by saying 
"Ran $G$ exists". Let us re-write $(i i)$ explicitly as:

(iii) for each $B \in B, B\left(B,\left(\operatorname{Ran}_{K} G\right)-\right)$ with $B\left(B, \varepsilon_{-}\right)$is the right Kan extension of $B\left(B, G_{-}\right)$along $K$.

In detail, (iii) means that for each $B \in B$ and for each $V$-functor $Q: D \rightarrow V$, the function

$$
\phi: V_{-\operatorname{nat}}\left(Q, B\left(B,\left(\operatorname{Ran}_{K} G\right)-\right)\right)+V_{-\operatorname{nat}}(Q K, B(B, G-))
$$

is an isomorphism, where $\phi$ sends $B$ to the composite

$$
Q K \underset{B K}{\longrightarrow} B\left(B,\left(\operatorname{Ran}_{K} G\right) K-\right) \underset{B(B, \varepsilon-)}{\longrightarrow} B(B, G-) \text {. }
$$

Now in fact we can replace $(i)$ and $(i i)$ as the definition by (iii) alone, since

PROPOSITION 8.1. (iii) implies (i).

Proof. Given $P: D+B$ take $Q$ in (8.4) to be $B(B, P-)$. Then the U-Yoneda-Lemma, with [4], Chapter III, Proposition 7.9, turns (8.4) into $(8.2)$.

A further simplification is given by:

PROPOSITION 8.2. In order that the $\phi$ of (8.4) be an isomorphism for all $Q$, it suffices that it be so for all $Q$ of the form $D(D,-) \otimes X$, where $D \in D$ and $X \in V$.

Proof. Taking Theorem 3.1 along with Proposition 7.1 and Proposition 7.5 , and dualizing, we express an arbitrary $Q$ as $\int^{D} D(D,-) \otimes Q D$; the result follows easily.

This now yields the "formula" for pointwise right Kan extensions:

THEOREM 8.3. The pointwise right Koon extension $\left(\operatorname{Ran}_{K} G, \varepsilon\right)$ of $G: A \rightarrow B$ along $K: A \rightarrow D$ exists if and only if, for each $D \in D$, the mean cotensor product $\left(\{D(D, K-), G\}, \lambda_{D}\right)$ exists. We have

$$
\left(\operatorname{Ran}_{K} G\right) D=\{D(D, K-), G\}
$$

while $\varepsilon_{A}:\left(\operatorname{Ran}_{K} G\right) K A \rightarrow G A$ corresponds under the Yoneda isomorphism to 


$$
\lambda_{D, A}: D(D, K A)+B(\{D(D, K-), G\}, G A) .
$$

Proof. Proposition 8.2 allows us to replace $Q$ by $D(D,-) \otimes X$ in (8.4), which then transforms under the $V$-Yoneda-Lemma into an isomorphism (8.8) $\pi:{ }_{0}\left(X, B\left(B,\left(\operatorname{Ran}_{K} G\right) D\right)\right) \cong V_{-\operatorname{nat}}(D(D, K-),[X, B(B, G-)])$.

Comparing this with (3.2) gives the "only if" part. For the "if" part we have to use Proposition 7.4 to make the right side of (8.6) V-functorial in $D$; we leave the easy details to the reader.

By Theorem 7.2, it comes to the same thing (cf. [7]) to say that for each $D$ the $V$-functor of $B$ given by $\int_{A}[D(D, K A), B(B, G A)]$ exists and admits a representation

$$
B\left(B,\left(\operatorname{Ran}_{K} G\right) D\right) \cong \int_{A}[D(D, K A), B(B, G A)]
$$

The formula (8.6) contains as special cases the two classical ones: for arbitrary $V$ if $B$ is cotensored Proposition 7.1 allows us to write $(8.6)$ as

$$
\left(\operatorname{Ran}_{K} G\right) D=\int_{A}[D(D, K A), G A],
$$

$\operatorname{Ran}_{K} G$ existing if and only if the right side exists for all $D$. Again for $V=$ Set and arbitrary $B$, Theorem 5.3 allows us to replace $(8.6)$ by

$$
\left(\operatorname{Ran}_{K} G\right) D=\lim (D / K \underset{d}{A} \underset{G}{\rightarrow} B),
$$

$\operatorname{Ran}_{K} G$ again existing if-and only if the right side exists for all $D$. (In (8.11) we have replaced the comma category $I / D(D, K-)$ of Theorem 5.3 by the isomorphic comma category $D / K$, in which $D$ denotes the unique functor $I \rightarrow D$ with image $D \in D$.)

In one case pointwiseness is automatic:

PROPOSITION 8.4. If $B$ is tensored and $\operatorname{ran}_{K} G$ exists, it exists pointuise.

Proof. $B(B,-): B \rightarrow V$ has a left adjoint and therefore respects all right Kan extensions. 
Since only the pointwise case is of real interest, we want the correspondingly stronger notion of "respects". We say that $T: B \rightarrow C$ respects $\operatorname{Ran}_{K} G$ (as distinct from $\operatorname{ran}_{K}^{\prime} G$ ) if $T\left(\operatorname{Ran}_{K} G\right.$ ) with $T \varepsilon$ is $\operatorname{Ran}_{K} T G$ (and not just $\operatorname{ran}_{K} T G$ ). Since the representables $B(B,-)$ land in $V$, which is tensored, it follows from Proposition 8.4 that they actually respect $\operatorname{Ran}_{K} G$. In general, it is clear from (8.6) that $T$ respects $\operatorname{Ran}_{K} G$ if and only if it preserves $\{D(D, K-), G\}$ for each $D$.

The classical elementary results about pointwise right Kan extensions now follow without any hypothesis that $B$ is cotensored; the next few propositions give examples.

PROPOSITION 8.5. If $\operatorname{Ran}_{K} G$ exists and $K$ is fully faithful, $\varepsilon$ is an isomorphism; so that $\operatorname{Ran}_{K} G$ is indeed an "extension" of $G$.

Proof. By (8.6) we have $\left(\operatorname{Ran}_{K} G\right) K A=\{D(K A, K-), G\}$; since $K$ is fully faithful as a $V$-functor, this is isomorphic to $\{A(A,-), G\}$, which is $G A$ by Theorem 3.1 .

We call $K$ codense if the canonical map

$$
D(B, D) \rightarrow \int_{A}[D(D, K A), D(B, K A)]
$$

is an isomorphism for all $B, D \in D$.

PROPOSITION 8.6. To say that $K$ is codense is to say that $\{D(D, K-), K\}=D$ for all $D$, or equally to say that $\operatorname{Ran}_{K} K$ exists and is the identity $1: 0 \rightarrow 0$.

PROPOSITION 8.7. If $K: A \rightarrow D$ has a left adjoint $L: D \rightarrow A$ then $\operatorname{Ran}_{K} G$ exists and is given by $G L$, while $\varepsilon: G L K+G$ comes from the counit of the adjunction. In this case $\operatorname{Ran}_{K} G$ is respected by any $T: B \rightarrow C$ whatever.

Proof. The $\left\{D\left(D, K_{-}\right), G\right\}$ of $(8.6)$ becomes $\{A(L D,-), G\}$, which is GLD by Theorem 3.1. The last statement of the proposition is evident. Our Theorem 6.5 translates into:

PROPOSITION 8.8. $G: A \rightarrow B$ has a left adjoint if and only if 
$\operatorname{Ran}_{G} \mathcal{I}$ exists and is respected by $G$, where $I$ is the identity $A \rightarrow A$.

In fact the same is true ([3], Theorem I.4.1) with ran $G^{1}$ replacing $\operatorname{Ran}_{G^{1}}$. By Proposition 8.7, however, the extra strength of the latter result is only apparent.

\section{Functor categories}

On the existence of functor categories, we have nothing to add to the account in [2]; we get from $V$-categories $A$ and $B$ a $V$-category $[A, B]$ called the functor category if the end $\int_{A} B(T A, S A)$ exists for all $T, S: A \rightarrow B$; then this end is $[A, B](T, S)$. The underlying ordinary category $[A, B]_{0}$ is that of the $U$-functors from $A$ to $B$ and the $V$-natural transformations between them.

When $[A, V]$ exists we can write $(7.5)$ as

$$
B(B,\{F, G\}) \cong[A, V](F, B(B, G-)) ;
$$

when $\left[A^{o p}, V\right]$ exists we have the dual

$$
B(H \star G, B) \cong\left[A^{\mathrm{op}}, V\right](H, B(G-, B)) ;
$$

these formulae have clearly influenced our choice of notation.

When the functor categories $[A, V]$ and $[A, B]$ exist, we can take $F$ and $G$ themselves as the "extra variables" in Proposition 7.4 , so that $\{$,$\} becomes a V$-functor

$$
\{,\}:[A, V]^{\mathrm{OP}} \otimes[A, B] \rightarrow B \text {. }
$$

Again by Proposition 7.5 the isomorphism $\{A(A,-), G\} \cong G A$ of Theorem 3.1 is $V$-natural in both $A$ and $G$.

When $[A, V]$ exists write $Y: A \rightarrow[A, V]^{\text {Op }}$ for the Yoneda embedding sending $A$ to $\mathrm{A}(A,-)$. For $F: \mathrm{A} \rightarrow V$ the isomorphism $\{\mathrm{A}(A,-), F\} \cong F A$ becomes $[\mathrm{A}, U](Y A, F) \cong F A$, or $[\mathrm{A}, V]^{\mathrm{Op}}(F, Y A) \cong F A$.

PROPOSITION 9.1. Let $[A, V]$ exist, let $Y: A \rightarrow[A, V]^{\circ \mathrm{P}}$ be the Yoneda embedding, and let $G: A \rightarrow B$ be a $V$-functor. Then $\operatorname{Ran}_{Y} G$ exists 
if and only if $\{F, G\}$ exists for all $F \in[A, V]$; and

$$
\left(\operatorname{Ran}_{Y} G\right) F=\{F, G\} .
$$

Proof. By (8.6) we have $\left(\operatorname{Ran}_{Y} G\right) F=\left\{[A, V]^{\mathrm{op}}\left(F, Y_{-}\right), G\right\} ;$ and $[A, V]^{\mathrm{OP}}(F, Y-)=F$.

PROPOSITION 9.2. Let $[A, V]$ exist and let $Y: A \rightarrow[A, V]^{\text {op }}$ be the Yoneda embedding. For each $F \in[A, V]$ we have

$$
\{F, Y\}=F \text {. }
$$

Thus by the preceding proposition $\operatorname{Ran}_{Y} Y$ is the identity, or $Y$ is codense.

Proof. We use Theorem 7.2. We have

$$
\int_{A}\left[F A,[A, V]^{\mathrm{OP}}(T, Y A)\right]=\int_{A}[F A, T A]=[A, V]^{\mathrm{OP}}(T, F) .
$$

Finally we observe that Theorem 7.2 together with Proposition 7.4 gives, using the Fubini Theorem for ends:

PROPOSITION 9.3. Let $F: A \rightarrow V, G: A \rightarrow[K, B] ;$ and suppose that $G$ corresponds to $H: A \otimes K \rightarrow B$. If $\{F, H(-, K)\}$ exists for each $K$ then $\{F, G\}$ exists and $\{F, G\} K=\{F, B(-, K)\}$.

\section{Completeness and ordinary limits}

We call the $V$-category $B$ complete if $\{F, G\}$ exists for all $F: A \rightarrow V$ and $G: A \rightarrow B$ when $A$ is small. Since $I$ is small, this implies that $B$ is cotensored; and is equivalent to the assertion that $B$ is cotensored and admits all ends with small domain.

If $V$ is complete the functor category $[A, B]$ exists whenever $A$ is small. The converse is also true: if all functor categories with small domain exist, then $[A, V]$ exists and thus, by Proposition 7.1, $\{F, G\}$ exists for $F, G: A \rightarrow V$.

It is clear from (8.6) that, for $K: A \rightarrow D$ and $G: A \rightarrow B$, $\operatorname{Ran}_{K} G$ exists if $A$ is small and $B$ is complete. If $V$ is complete, so that $[A, V]$ exists for small $A$, Proposition 9.1 provides a converse: $B$ is 
complete if and only if $\operatorname{Ran}_{Y} G$ exists for all $G: A+B$ with $A$ small, $Y$ being the Yoneda embedding $A \rightarrow[A, V]^{\mathrm{Op}}$.

To analyze completeness - that is, the existence of $\{F, G\}$ - in more familiar terms, we turn to the question of ordinary limits in $B$, as adumbrated in $\$ 1$. We have left these until last; for one thing they do not arise until we mix ordinary categories with our $V$-categories, and for another they do not come as a special case of $\{F, G\}$ without a mild hypothesis on $V$.

Suppose we have an ordinary category $K$, a $V$-category $B$, and ordinary functors $F: K \rightarrow V_{0}$ and $G: K \rightarrow B_{0}$. We modify the definition of $\$ 3$ to define the mean cotensor product $\{F, G\}^{\prime}$ of $F$ and $G$; it is an object $\{F, G\}^{\prime}$ of $B$ together with a (merely) natural transformation $\lambda: F \rightarrow B\left(\{F, G\}^{\prime}, G-\right)$ such that the function

$$
\pi: V_{0}\left(X, B\left(B,\{F, G\}^{\prime}\right)\right) \rightarrow \operatorname{nat}(F,[X, B(B, G-)])
$$

is an isomorphism, where $\pi$ still sends $f$ to the composite (3.3).

The reader will easily verify that virtually all we have said carries over to this case without change. We still get the cotensor product by taking $K$ to be the unit (ordinary!) category $I$; we still have weak and strong existence; we still have Theorems 6.1 and 6.2 ; we can define ends in $B$ of an ordinary bifunctor into $B_{0}$; we can define the $V$-functor $\operatorname{Ran}_{K} G$ from the ordinary functors $K$ and $G$ by using (8.6) as a definition; and the ordinary functors $K \rightarrow B_{0}$ form a V-category $[K, B]$ (if the appropriate ends exist), whose underlying ordinary category $[K, B]_{0}$ is the ordinary functor-category $\left[K, B_{0}\right]$. The theorems that are lacking are those involving the representable $\mathrm{A}(A,-)$, which we no longer have.

of course in the case $V=$ Set there is no difference between $\{F, G\}^{\prime}$ and $\{F, G\}$ - except that the latter is defined only for Setcategories $K$, that is, only for locally small ordinary categories $K$.

We can now express in terms of $\{,\}^{\prime}$ the notion of the limit in $B$ (as distinct from the limit in $B_{0}$ ) of an ordinary functor $G: K \rightarrow B_{0}$, 
as discussed in 51 . We write $\operatorname{Lim} G$ for the limit in $B$, retaining $\lim G$ for the mere limit in $B_{0}$.

THEOREM 10.1. Denote by $*: K \rightarrow V_{0}$ the constant fronctor at $I$. Then for $G: K \rightarrow B_{0}$ we have
$\operatorname{Lim} G=\{*, G\}^{\prime}$,

either side existing if the other does. We also have

$\lim G=\{\star, G\}^{\prime}$,

the left side existing precisely when the right side exists weakly.

Proof. For the second statement, observe that the codomain of (3.4), with $V$-nat now replaced by nat, may be identified with the set of cones of vertex $B$ over $G$. The first statement follows then by (the analogue of) Proposition 5.4 .

Suppose now that $v_{0}$ admits small copowers; in practice this is an extremely mild condition, for the base category $V$ is usually highly respectable; if it were not, one would anticipate some untidiness in the notion of completeness for $V$-categories. Then on any locally-small ordinary category $K$ we can form the free $V$-category $A$, with the same objects as $K$, by taking for $\mathrm{A}(A, B)$ the $K(A, B)$-th copower of $I$. There is now a bijection between ordinary functors $G: K+B_{0}$ and $V$-functors $\hat{G}: A \rightarrow B$; and we have

$$
\{F, G\}^{\prime}=\{\hat{F}, \hat{G}\},
$$

so that we do not need $\{F, G\}^{\prime}$ as a separate notion after all. In particular we have

$$
\operatorname{Lim} G=\{*, \hat{G}\},
$$

where now * :A $\rightarrow$ is the constant $V$-functor at $I$ induced by * : $K \rightarrow V_{0}$. This justifies our claim that all hitherto-used limit notions for $U$-categories are subsumed under our notion $\{F, G\}$ of mean cotensor product.

Splitting up the existence of limits in $B$ into their existence in $B_{0}$ and their preservation by the representables $B(B,-): B_{0}+V_{0}$, we 
have

THEOREM 10.2. For a V-category B, consider the assertions

(a) $B$ is complete in the above sense that all $\{F, G\}$ exist for small A;

(b) $B$ is cotensored, $B_{0}$ admits small limits, and each $B(B,-)$ preserves them.

Then (b) implies (a), while (a) implies (b) if $v_{0}$ admits small copowers.

When $B$ is tensored, (b) can be replaced by

(b') $B$ is cotensored and $B_{0}$ admits small limits.

When $B$ is $V$ itself, (b) can be replaced by

(b") $V_{0}$ admits small limits.

Finally in the case $V=$ Set, $B$ is complete if and only if it admits small limits.

Proof. We have seen that $B$ is complete if and only if it admits cotensor products and small ends. That the latter exist under the hypotheses (b) is proved in [2],3.3. When $v_{0}$ admits small copowers, the converse implication $(a) \Rightarrow(b)$ follows from (10.4). Proposition 5.1 allows the reduction of $(b)$ to $\left(b^{\prime}\right)$, while the further reduction to $\left(b^{\prime \prime}\right)$ is trivial since $V$ is cotensored. The final remark about the case $V=$ set follows from (10.2) and Theorem 5.3 .

The usual definition of completeness has been the slightly stronger (b); the case where $v_{0}$ does not admit small copowers is too rare to Justify an attempt to introduce separate names for $(a)$ and $(b)$ in this general account.

With the remark that preservation of $\{*, G\}^{\prime}$ by $T$ is precisely what is normally meant by preservation of $\operatorname{Lim} G$ by $T$, we leave it to the reader to formulate for preservation by $T$ the analogue of Theorem 10.2. 


\section{References}

[1] Brian Day, "On closed categories of functors", Reports of the Mictuest Category Seminar IV, 1-38 (Lecture Notes in Mathematics, 137. Springer-Verlag, Berlin, Heidelberg, New York, 1970).

[2] B.J. Day and G.M. Kelly, "Enriched functor categories", Reports of the Midwest Category Seminar III, 178-191 (Lecture Notes in Mathematics, 106. Springer-Verlag, Berlin, Heidelberg, New York, 1969).

[3] Eduardo J. Dubuc, Kan extensions in enriched category theory (Lecture Notes in Mathematics, 145. Springer-Verlag, Berlin, Heidelberg, New York, 1970).

[4] Samuel Eilenberg and G. Max Kelly, "Closed categories", Proc. Conf. Categorical Algebra, La Jolla 1965, $421-562$ (Springer-Verlag, Berlin, Heidelberg, New York, 1966).

[5] G.M. Kelly, "Adjunction for enriched categories", Reports of the Midwest Category Seminar III, 166-177 (Lecture Notes in Mathematics, 106. Springer-Verlag, Berlin, Heidelberg, New York, $1969)$.

[6] S. Mac Lane, Categories for the working mathematician (Graduate Texts in Mathematics, 5. Springer-Verlag, New York, Heidelberg, Berlin, 1971).

[7] M. Zandarin-Vandenbeyvanghe, "Extension de Kan relative", Séminaires de Mathématique Pure, Rapport $n^{\circ} 39$ (Institut de Mathématique Pure et Appliquée, Université Catholique de Louvain, Louvain-laNeuve, 1973).

Département de Mathématiques,

Université de Louvain, Louva in-la-Neuve,

Belgium;

Department of Pure Mathematics, University of Sydney, Sydney, New South Wales. 